\title{
A New Routing Technique Based on Fuzzy Logic for Multi-Hop Wireless Mesh Networks
}

\author{
Er. Pushpender, Prof. (Dr.) Sohan Garg \\ ${ }^{1}$ ResearchScholar, ShriVenkateshwara University, Gajraula(UP), India \\ ${ }^{2}$ Professor, C.C.S. University, Meerut (UP), India,
}

\begin{abstract}
WMN technology is different from traditional wireless networks due to its several characteristics such as increased reliability, low installation costs, large coverage area, automatic network connectivity etc. WMN is multi-hop network consisting of routers and gateways and mobile nodes. Routing in such a heterogeneous environment has put enormous challenges to participating nodes and routers to deliver packet from one corner to other. The difficulty in managing or controlling the link quality and reliability in wireless mesh networks makes it very hard to find and maintain a good and stable path from the source to the destination. Also, the wireless medium is broadcast in nature, transmission on one link may interfere with the transmissions on the neighboring links. This broadcast medium contention brings fundamental constraints on the routing performance, such as throughput, delay, mobility, bandwidth etc. It is very challenging to achieve optimal routing performance. In WMN, any node may compromise the routing protocol functionality by disrupting the route discovery process. In this paper, we understand the various routing problems related to throughput, bandwidth, and mobility. In this paper, we have proposed a new routing technique that is totally network dependent and will remove all the routing problems in WMNs.
\end{abstract}

Keywords: - Bandwidth, Mobility, Wireless Mesh Networks, Mobile Ad hoc network, topology, AODV, DSR

\section{INTRODECTION}

A Wireless Mesh Network (WMN) is based on ad-hoc networks, where each node transfers data to and from an Access Point (AP) which is connected to the Internet by a wired or wireless network. These AP need not be in the reach of all the nodes in the network. Nodes around the AP forward the packets from the faraway nodes to the AP. If there are a significant number of nodes in the network, faraway nodes can transfer data with the AP in a few hops. Besides mobility, WMN have the advantages [10] viz., they can work in a decentralized fashion, are cheap with minimum investment for initial infrastructure, more reliable, scalable and provide increased coverage. They are widely used in campus networks, metropolitan area networks, transportation system, security surveillance system, etc. Thus, they eliminate the drawbacks predominant in a traditional network which uses a wired connectivity to a base station, wherein every user connects it through a point to multi-point protocol [11]. Instead of using WMN, few access points can be setup which can schedule the medium usage scheduling for the different users in the network. Users may use different routes based on the routing protocol. For streaming of audio and video information, a feasible path is to be chosen based on multiple constraints. This is called multi constraint routing. The constraints can be for a link and/or for a path. In the case of a link, the constraints specify the restrictions for a single link whereas for a path, the constraints are for the entire path (end to end). In addition to this, other issues such as routing for multicast applications, scalability of routing protocols, cross-layer design between routing and MAC protocols are also under study [12]. This could become a cheap and simple alternative to wired telephone and cable networks. But there are many important issues[12] such as, integrating multiple performance metrics into a routing protocol to achieve an optimal overall performance, scalability of routing protocols, routing for multicast applications, and cross-layer design between routing and MAC protocols.

On the other hand, wireless routers in WMNs are expected to be unstable. Apart from routers, wireless links can be unstable. The quality of data transmission can be weakened by multi-path fading effects, external interference and weather conditions. The potential instability of routers and links can appear after determining the optimal route, during transmission of data, which brings us additional requirements related to the protocol. Existing routing protocols applied to WMNs do not have enough scalability [1,2]. Some protocols perform an additional check after finding an optimal route, but it is certain that the conditions can changed over time [2]. Usually, the changes are detected only when the link fails or if some of the routers do not respond $[3,4]$. The authors of $[5,6]$ suggested some solutions, where the route testing is done periodically, but the testing interval is not small enough to follow current network monitoring. Besides, the protocol should obtain adaptability in a sense of topology change, as well as adaptability of routers and links, which is quite often in WMNs. In addition to the problem of defining the optimal routing protocol, while designing the WMN, the problem of the quality of 
wireless transmissions has to be considered. Several authors have provided suggestions for solving the transmission problem and making optimal use of the available spectrum [7-9].

In this paper, one solution for optimal routing in WMNs is proposed, more specifically, the one emphasizing the management of network resources. Taking into consideration all particularities of WMNs, the proposed solution provides, in the Pareto sense, the optimal route selection, in the shortest time interval, taking into account the current state of the network and optimal use of all network resources. In that way, it is necessary to choose the route in accordance with the needs of future subscribers and their traffic, with as small as possible number of rerouting and the optimal loading balance of the entire network.

\section{LITERATURE SURVEY}

In a network like the WMN, the various constraints like collisions, traffic level, buffer occupancy, battery power, etc. need to be considered. It is not enough if only one constraint is considered. This is because of the complex relationship existing between the different constraints. Multi-constrained routing is a NP complete problem and does not have a polynomial solution. It is required to use various heuristics and soft computing techniques to solve them [13]. A Fuzzy system is best suited in making optimal routing decisions in a network involving multiple constraints and multiple objectives. There are several studies of fuzzy multi-objective routing where a fuzzy system is implemented over classical methods like DSR to do multi-objective routing. A fuzzy system is considered over classical DSR in [14]. Routes are decided based on the metrics Node Delay, Node loss and node speed. A fuzzy routing algorithm based on several metrics for a mobile ad-hoc network is proposed in [15].

A fuzzy logic system where unnecessary routes are eliminated by removing links not accepted by the system is considered in [16]. An adaptive algorithm based on fuzzy logic to change the security level of the mobile node is proposed in [17].

In this paper, we consider a fuzzy system for making routing decisions in WMN where the destination AP is common for several users. Here it is necessary that the traffic gets spread across the system for maximum bandwidth usage. Various constraints that are considered are buffer occupancy, residual energy of nodes and the distance of source (hops) from the AP.

\section{THE PROPOSED ROUTING TECHNIQUE}

Here Fuzzy Logic has been used for routing and management of a Wireless Mesh Network. The proposed fuzzy logic based routing algorithm takes into account of three input variables, throughput and mobility and bandwidth. The absolute value of each of these parameters can take a large range at different points on the network. We have considered the normalized values for each parameter. Now, 'crisp' normalized values are being converted into fuzzy variables. For this, three fuzzy sets have been defined for each variable [18]. The sets, low (from 0 to 0.4 ), medium (from 0.2 to 0.8 ) and high (from 0.6 to 1.0) have been used for the input variable throughput (figure 1) and the sets, poor (from 0 to 0.4 ), average (from 0.2 to 0.8 ) and excellent (from 0.6 to 1.0) have been used for input variable bandwidth (figure 2) and the sets, low (from 0 to 0.4 ), medium (from 0.2 to 0.8 ) and high (from 0.6 to 1.0 ) have been used for the input variable mobility (figure 3 ). The normalized value of each parameter is mapped into the fine sets. Each value will have some grade of membership function for each set. The memberships that have been defined for each of the fuzzy set for any particular input variable are triangular in shape. Next the rules of inference have been written. Initially total 27 rules were devised. The crisp value of input variable was given and a defuzzified crisp value for selected variable was calculated from the derived algorithm. An output linguistic variable is used to represent the route. Proposed optimal routes are based upon the fuzzy rules for different ranges of the metric availability [18]. The routes (figure 4) are defined as below optimal (from 0 to 0.4 ), suboptimal (from 0.2 to 0.8 ) and optimal (from 0.6 to 1.0 ) between two mobile hosts. The below optimal indicates not optimal path, the sub optimal indicates good path and the optimal path indicates the best path [18]. The proposed routing algorithm can apply to different routing metrics. These routes have to satisfy the mobility, throughput and bandwidth requirements of the network. The grade of membership function can be any where between 0 and 1 for each fuzzy set. The defuzzified crisp value for selected variable was calculated from the derived algorithm. The proposed Fuzzy Logic \& bandwidth based routing algorithm for Wireless Mesh Network is classified as "A new Routing Technique based on fuzzy logic for multi-hop Wireless Mesh Networks".

Our problem is to find the optimal and suitable route from source to the destination based on mobility, throughput and bandwidth. The system is based on the fuzzy inference system. The major components of the system consist of the knowledge base, decision making, fuzzification and defuzzification. Now we will write the fuzzy rules based on the mobility, throughput and bandwidth and try to find out the optimal path or route. 


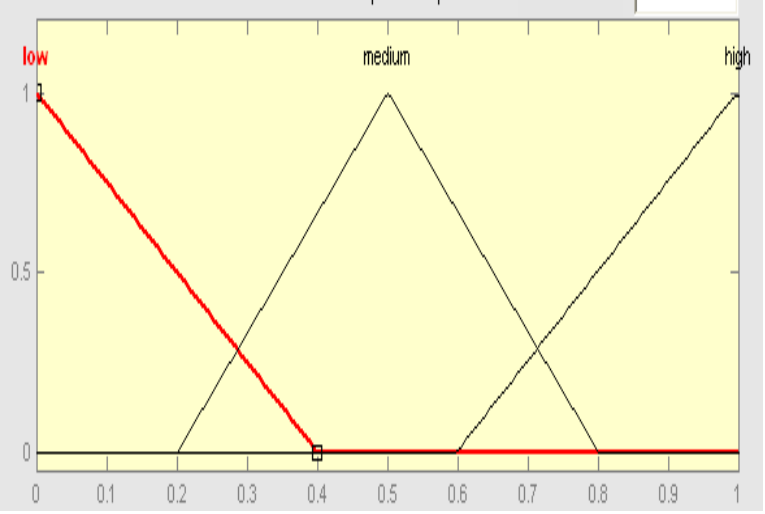

Figure 1. Input Variable 'Throughput'

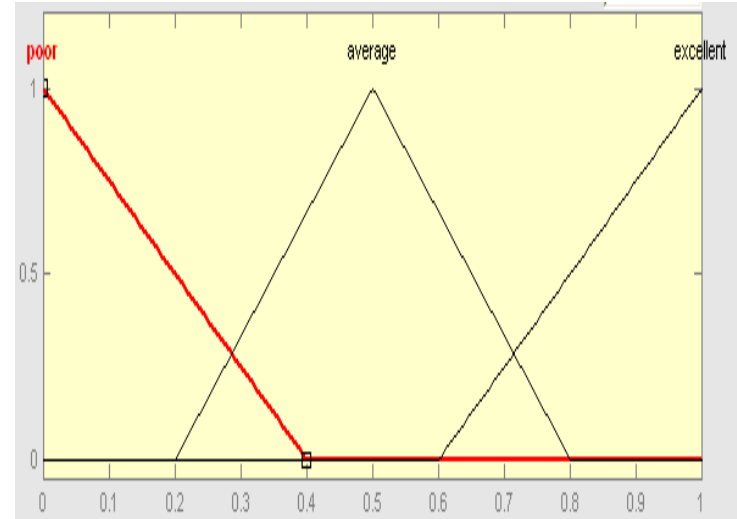

Figure 2. Input Variable 'Bandwidth'

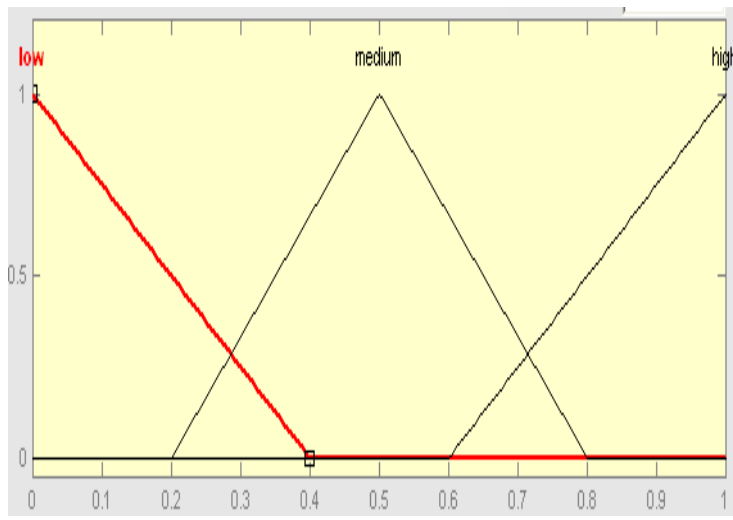

Figure 3. Input Variable 'Mobility'

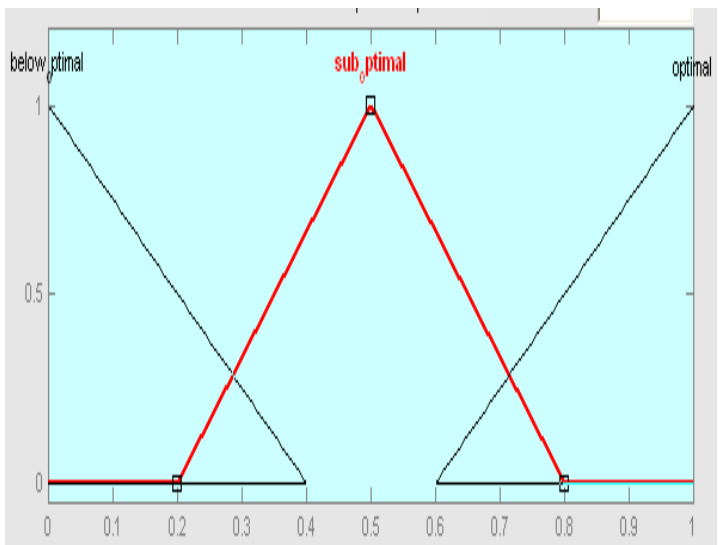

Figure 4. Output Variable 'Route' 
The Fuzzy Inference rules for the proposed routing technique are:

Table 1: Inference Rules

\begin{tabular}{|c|c|c|c|c|}
\hline $\begin{array}{l}\text { Rule } \\
\text { No. }\end{array}$ & Throughput & Mobility & Bandwidth & Route \\
\hline 1 & low & low & poor & below optimal \\
\hline 2 & low & low & average & below optimal \\
\hline 3 & low & low & excellent & sub optimal \\
\hline 4 & low & medium & poor & below optimal \\
\hline 5 & low & medium & excellent & sub optimal \\
\hline 6 & low & medium & excellent & sub optimal \\
\hline 7 & low & high & poor & below optimal \\
\hline 8 & low & high & average & below optimal \\
\hline 9 & low & high & excellent & suboptimal \\
\hline 10 & medium & low & average & sub optimal \\
\hline 11 & medium & low & average & optimal \\
\hline 12 & medium & low & excellent & optimal \\
\hline 13 & medium & medium & poor & below optimal \\
\hline 14 & medium & medium & average & sub optimal \\
\hline 15 & medium & medium & excellent & optimal \\
\hline 16 & medium & high & poor & below optimal \\
\hline 17 & medium & high & average & sub optimal \\
\hline 18 & medium & high & excellent & optimal \\
\hline 19 & high & low & poor & sub optimal \\
\hline 20 & high & low & average & optimal \\
\hline 21 & high & low & excellent & optimal \\
\hline 22 & high & medium & poor & sub optimal \\
\hline 23 & high & medium & average & sub optimal \\
\hline 24 & high & medium & excellent & optimal \\
\hline 25 & high & high & poor & below optimal \\
\hline 26 & high & high & average & sub optimal \\
\hline 27 & high & high & excellent & optimal \\
\hline
\end{tabular}

The routes based upon the above rules have been shown with the help of the following graphs:

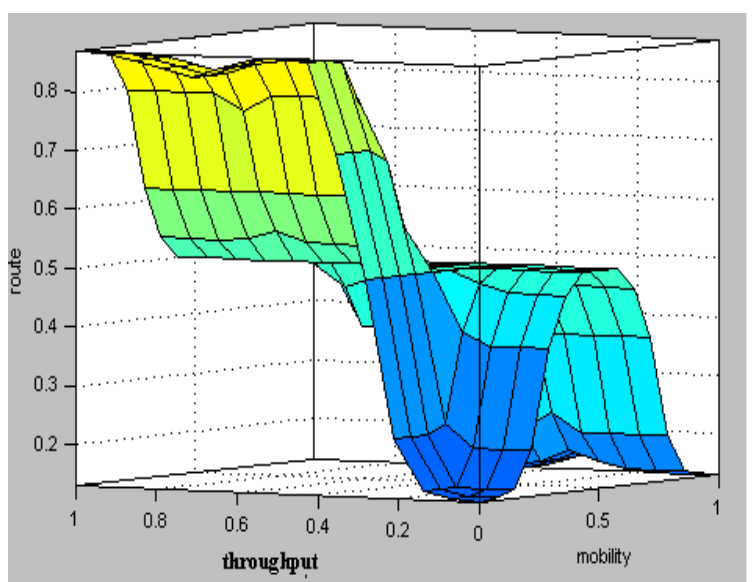

Figure 5. 'Route' O/P w.r.t. 'Mobility' and 'Throughput'

In figure 5 throughput and mobility are the fuzzy input variable for the proposed routing algorithm which lies on the horizontal axes and route is the output variable which has been shown on the vertical axis. It is very clear from the figure that at constant throughput and low mobility the Route is below optimal, but for any value of the throughput if we increase the mobility then the route also increases and becomes sub-optimal. Finally at high mobility the route will be below optimal for constant throughput. 


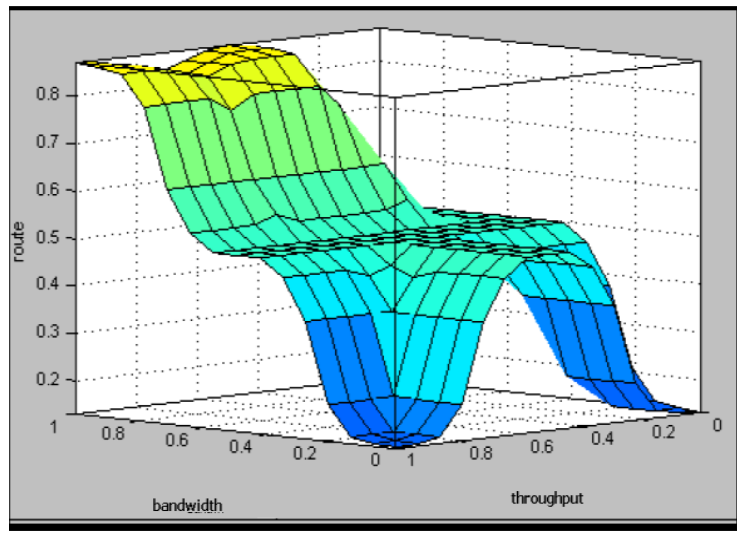

Figure 6 'Route' O/P w.r.t. 'Signal Power' and 'Bandwidth'

In figure 6 the inputs of the algorithm (throughput and bandwidth) are on the horizontal axes and the output (route) is on the vertical axis. According to the figure It is clear that at constant bandwidth and low throughput the route is below optimal but if the throughput is increased up to medium the route is sub optimal for poor bandwidth. In the same way at high throughput and poor bandwidth the route becomes below optimal. If delay is increased up to average at high throughput then the route is suboptimal but route becomes optimal at excellent bandwidth and at high throughput.

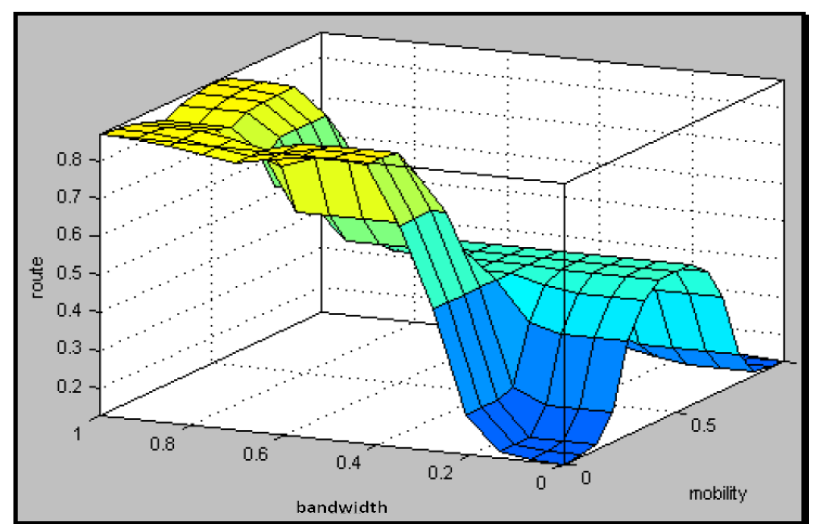

Figure 7. 'Route' O/P w.r.t. 'Mobility' and 'Throughput'

In figure 7 bandwidth and mobility are the input variables and route is the output variable. The $3 \mathrm{D}$ decision surface illustrate that route is below optimal for poor bandwidth and low mobility. As the mobility is increased up to medium the route will be sub optimal. Again the similar process occurs and route is below optimal for poor bandwidth. But as the bandwidth becomes high, route increases up to sub optimality and optimality when the mobility is medium and high respectively.

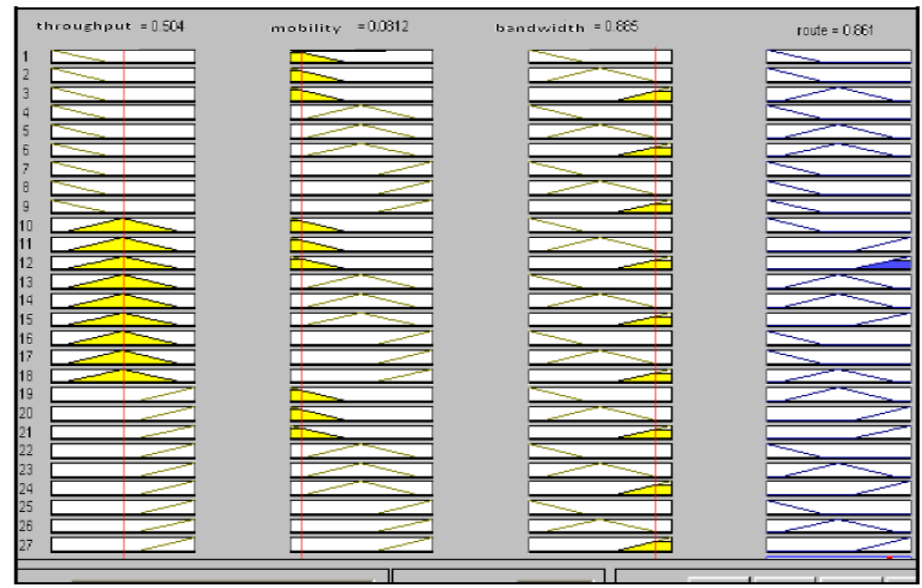

Figure 8. 'Route' O/P w.r.t. Medium 'Throughput', Low 'Mobility' and Excellent 'Bandwidth' 
The figure 8 illustrates that when throughput is medium (0.504), mobility is low (0.0812) and bandwidth is excellent $(0.885)$ then in this condition the route is optimal $(0.861)$. So this algorithm works well when mobility is low and throughput is medium.

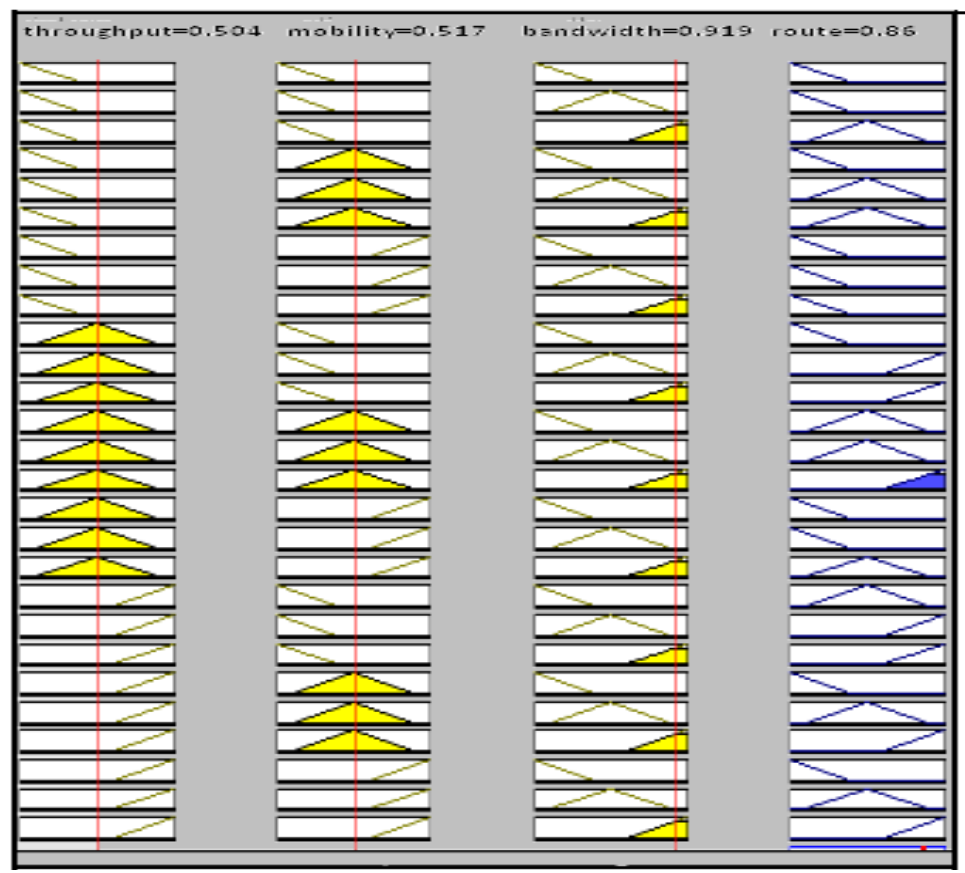

Figure 9. 'Route' O/P w.r.t. Medium 'Throughput', Medium 'Mobility' and Excellent 'Bandwidth'

In figure 9 we have seen that route is optimal at low mobility, medium throughput and at excellent bandwidth but against it when we increase the mobility towards medium (0.517) at medium throughput $(0.504)$ and excellent bandwidth $(0.919)$ the route is again optimal. So this algorithm works well at medium mobility also.

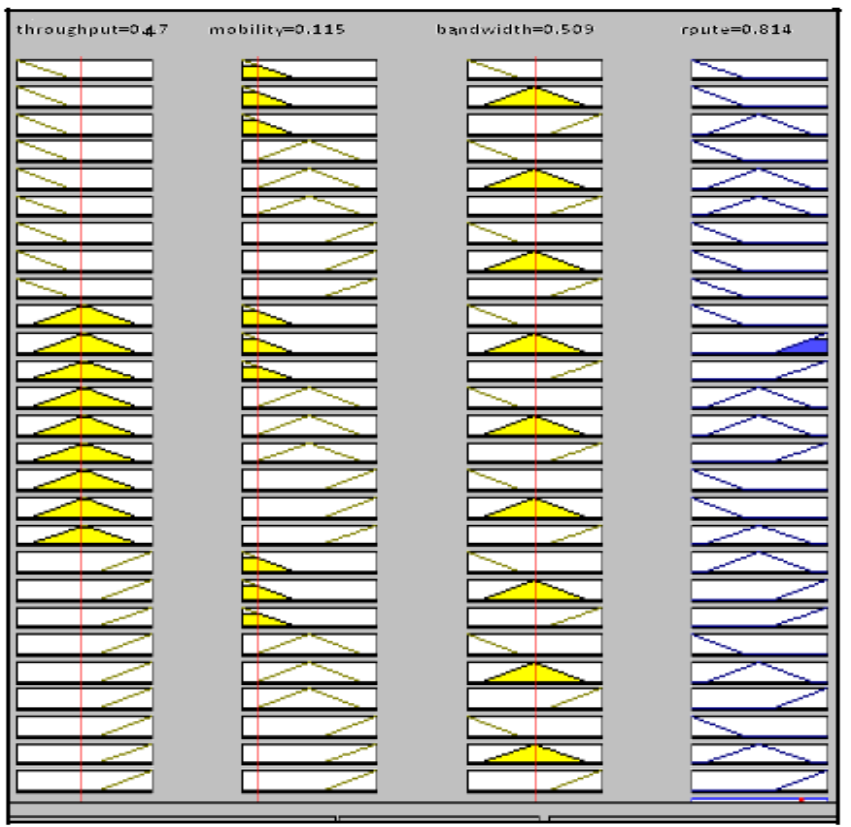

Figure 10. 'Route' O/P w.r.t. Medium 'Throughput', Low 'Mobility' and Average 'Bandwidth'

This is very clear from the figure $\mathbf{1 0}$ that in this protocol the value of the throughput (0.47) and mobility $(0.115)$ is same as in figure 6.8 but the difference is that when we will decrease the bandwidth up to average $(0.509)$ then the route is still optimal, but as we will increase the mobility then the route will be sub optimal. 


\section{SIMULATION RESULTS}

In decision surface (figure 5) the input variables throughput and mobility lie on horizontal axes and the output variable route lies on vertical axis. It shows that at constant throughput the route is below optimal for low mobility. But any throughput if we increase the mobility the route also starts increasing and becomes sub optimal. Finally when the mobility becomes high, the route will be below optimal for constant throughput. Now as we increase the throughput the route starts increasing towards optimality and becomes optimal for low and medium mobility. In figure 6 the inputs of the algorithm are on the horizontal axes and the output of the algorithm is on the vertical axis. Here, with the help of 3D surface we observe that at constant bandwidth and low throughput the route is below optimal but if the throughput is increased up to medium the route is sub optimal for poor bandwidth. Similarly at high throughput and poor bandwidth the route becomes below optimal. It can also be observed that with average bandwidth and high throughput the route is suboptimal and if we increase bandwidth up to excellent the route is optimal at high throughput. In figure 7 the input variables mobility and bandwidth lie at horizontal axes and the route at vertical axis. Here, we see that for poor bandwidth and low mobility the route is below optimal. As the mobility increases and arrive up to medium the route will be sub optimal. Again the similar phenomenon occurs and route is below optimal for poor bandwidth. But as the bandwidth becomes high, route increases up to sub optimality and optimality when mobility is medium and high respectively. In figure 8 we see that at medium throughput (0.504), low mobility (0.0812) and excellent bandwidth $(0.885)$ the route is optimal $(0.86)$ and as we increase the only mobility towards medium $(0.517)$ then the route is again optimal $(0.865)$ which has been shown in the figure 9 . In figure 10 , with medium throughput (0.47), low mobility $(0.115)$ and average bandwidth (0.509) the route becomes optimal again but as we increase the mobility then route becomes suboptimal.

\section{CONCLUSIONS}

One of the most important factors influencing performance of wireless mesh network is the routing protocol used. Existing protocols such as traffic balancing select the routes based on its usage and AODV chooses routes based on their length. To maximize the performance, and to reduce the overhead of the entire network, we have proposed our new routing technique based on throughput, mobility and bandwidth. After fully describing its functions and mechanism, we have suggested various optimizations to the protocol and utilized the concept of stability index. Experimental simulation results using MATLab shows that our routing technique is functional and effective. We do see the need in further experimentation in order to accurately access the practical effectiveness of our routing technique in a medium to large size network.

\section{REFERENCES}

[1] Haque, I.T. Randomized Routing Algorithms in Mobile Ad Hoc Networks; VDM Verlag: Saarbrücken, Germany, 2009.

[2] Ramachandran, K.N.; Sheriff, I.; Belding, E.M.; Almeroth, K.C. Routing stability in static wireless mesh networks. Lect. Notes Comput. Sci. 2007, 4427/2007, 73-82.

[3] Clausen, T.; Jacquet, P. Optimized link state routing protocol, Internet Engineering Task Force.Request for Comments 3626, October 2003.

[4] Perkins, C. Ad-hoc On-demand Distance Vector Routing. In Proceedings of the MILCOM, Orlando, FL, USA, 2-5 November 1997.

[5] Bicket, J.; Aguayo, D.; Biswas, S.; Morris, R. Architecture and Evaluation of an Unplanned 802.11b Mesh Network; ACM MobiCom: Cologne, Germany, 2005.

[6] Draves, R.; Padhye, J.; Zill, B. Routing in Multi-Radio, Multi-Hop Wireless Mesh Networks; ACM MobiCom: Philadelphia, PA, USA, 2004.

[7] Kusume, K.; Vilzmann, R.; Müller, A.; Hartmann, C.; Bauch, G. Medium access in spread-spectrum ad hoc networks with multiuser detection. EURASIP J. Adv. Signal Process 2009, doi:10.1155/2009/156247.

[8] Romaszko, S.; Blondia, C. Cross Layer PHY-MAC protocol for wireless static and mobile ad hoc networks. EURASIP J. Adv. Signal Process. 2009, doi:10. 1155/2009/278041.

[9] Chen, X.; Zhao, Z.; Jiang, T.; Grace, D.; Zhang, H. Intercluster connection in cognitive wireless mesh networks based on intelligent network coding. EURASIP J. Adv. Signal Process. 2009, doi:10.1155/2009/141097.

[10] Mihail L., "Wireless Mesh Networks: Opportunities and Challenges," in Proceedings of the Wireless World Congress, CA, pp. 1-6, 2005.

[11] Ian F., Xudong W., and Weilin W., "Wireless Mesh Networks: A Survey," Computer Journal of Networks and ISDN System, vol. 47, no. 4, pp. 445-487, 2005.

[12] Karol K. and Mark D., "Why are there so Many Routing Protocols for Wireless Mesh Networks?," in Proceedings of Irish Signal and Systems Conference, Dublin, pp. 1-5, 2006. 
[13] Korkmaz T. and Krunz M., "Bandwidth-Delay Constrained Path Selection Under Inaccurate State Information," IEEE ACM Transactions on Networking, vol. 2, no. 3, pp. 384-398, 2003.

[14] Wong Y. and Wong W., "A Fuzzy-Decision- Based Routing Protocol for Mobile Ad-Hoc Networks," in Proceedings of 10th IEEE International Conference on Networks, Singapore, pp. 317-322, 2002.

[15] Shivanajay M., Dipti S., and Chen T., "Evolutionary Fuzzy Multi-Objective Routing for Wireless Mobile Ad-Hoc Networks," in Proceedings of Congress on Evolutionary Computation, Singapore, pp. 19641971, 2004.

[16] Susan R. and Dirk P., "Multi-Metric Routing Decisions for Ad Hoc Networks Using Fuzzy Logic," in Proceedings of 1st International Symposium on Wireless Communication Systems, USA, pp. 403- 407, 2004.

[17] Jing N., Jiangchua W., Ji L., Xin H., and Zheng Z., "An Adaptive Fuzzy Logic Based Secure Routing Protocol in Mobile Ad-Hoc Networks," Computer of Journal Fuzzy Sets and Systems, vol. 157, no. 12, pp. 1704-1712, 2006.

[18] Sohan Garg, Payal ,Kansal, Viksit Kumar Sharma: “A Fuzzy Logic based network Dependant Routing Algorithm for Ad Hoc Wireles Network", Int. Journal Engg. Research and Application (IJERA), vol. 1 (1), pp. $1-10$

\section{About the Author's}

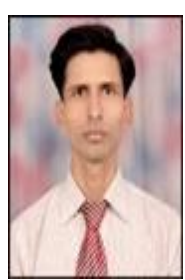

Er. Pushpender received the B.tech degree in Information Technology Engineering and M.Tech degree in Computer Science and Engineering from M.D. University, Rohtak (India). He has been in teaching profession more than three years. Beside he has the good industrial exposure in the field of computer technology and network. He is currently working as an Assistant Professor in the department of Computer Science and Engineering at S (PG) ITM, Rewari, India. Also he is a research scholar pursuing his Ph.D from Shri Venkateshwara University, Gajraula (UP), India in Wireless Mesh Network specializing Routing Protocols.

Dr. Sohan Garg: Presently working as Director, Sir Chhotu Ram Institute of Engineering and Technology, CCS University Campus Meerut, UP (India). He has worked as Director, IIMT Management College, Meerut, (UP), India. He received the Ph.D. degree in Computer Science from Institute of Advanced Studies, C.C.S. University Campus Meerut. He received the M.Tech degree in Computer Science from Manav Bharti University, Shimla H.P (India). He has published several research papers in National and international journals in his credit. He is also the guide of research scholar for almost twelve of Universities. 